\title{
The two sets of three semifields associated with a semifield flock
}

go back

full screen

close

quit

\author{
Michel Lavrauw*
}

\begin{abstract}
In 1965 Knuth [4] showed that from a given finite semifield one can construct further semifields manipulating the corresponding cubical array, and obtain in total six semifields from the given one. In the case of a rank two commutative semifield (the semifields corresponding to a semifield flock) these semifields have been investigated in [1], providing a geometric connection between these six semifields and it was shown that they give at most three non-isotopic semifields. However, there is another set of three semifields arising in a different way from a semifield flock, hence in total six semifields arise from a rank two commutative semifield (see [1]). In this article we give a geometrical link between these two sets of three semifields.
\end{abstract}

Keywords : semifields, translation planes, finite geometry

MSC 2000: 12K10, 51E15

\section{Introduction and motivation}

Throughout the article we will use the terminology and the notation from [1]. A semifield coordinatises a semifield plane, which corresponds to a semifield spread via the Andre-Bruck-Bose construction, see [3, Section 3.1]. A flock of a quadratic cone gives rise to a line spread of three-dimensional projective space (and hence to a translation plane) via the Thas-Walker construction, see [1], [6]. In case the flock is a semifield flock, the resulting translation plane is a semifield plane.

\footnotetext{
${ }^{*}$ This research has been supported by a VENI grant, part of the Innovational Research Incentives Scheme of the Netherlands Organisation for Scientific Research (NWO).
} 



As in Section 2 let $\mathcal{S}$ denote the semifield with multiplication

$$
(x, y) \circ(u, v)=(u x+y f(v), x v+y u+y g(v)) .
$$

Dualising the plane we get the semifield $\mathcal{S}^{*}$ by reversing the multiplication, i.e.,

$$
(x, y) \circ^{*}(u, v)=(x u+v f(y), u y+x v+v g(y)) .
$$

Both multiplications have $(1,0)$ as identity element. In order to obtain the multiplication for $\mathcal{S}^{* \dagger}$ we have to dualise the semifield spread obtained from $\mathcal{S}^{*}$ (see [1]). We have to find all $a, b, c, d \in \operatorname{GF}\left(q^{n}\right)$ for which

$$
\operatorname{tr}(x a+y b+(x u+f(y) v) c+(y u+x v+g(y) v) d)=0, \forall x, y \in \operatorname{GF}\left(q^{n}\right) .
$$

Putting $x=0$ we get the condition

$$
\operatorname{tr}\left(y b+f(y) v c+y u+g(y) v d=0, \forall y \in \mathrm{GF}\left(q^{n}\right) .\right.
$$

This implies $b=-(\hat{f}(v c)+u d+\hat{g}(v d))$. Similarly, after putting $y=0$ we get $a=-u c-v d$. Hence after some coordinate transformations, we get the multiplication

$$
(x, y) \cdot(u, v)=(x u+y v, u y+\hat{f}(x v)+\hat{g}(y v))
$$

In order for $(1,0)=(1,0) \cdot(1,0)$ to be the identity we have to define a new multiplication by $((x, y) \cdot(1,0)) \circ^{* \dagger}((1,0) \cdot(u, v))=(x, y) \cdot(u, v)$ (see [4]). We get

$$
(x, y) \circ^{* \dagger}(u, v)=\left(x u+y \hat{f}^{-1}(v), u y+\hat{f}\left(x \hat{f}^{-1}(v)\right)+\hat{g}\left(y \hat{f}^{-1}(v)\right)\right) .
$$

That $\hat{f}^{-1}$ is well defined follows from the fact that the multiplication o from the previous section has no zero divisors. In the previous we had the following multiplication for $\hat{\mathcal{S}}$ :

$$
(x, y) \hat{o}(u, v)=(x u+y v, x v+y \hat{f}(u)-y \hat{g}(v)) .
$$

We see that $(1,0) \hat{o}(u, v)=(u, v)$ and, $(x, y) \hat{o}(1,0)=(x, y \hat{f}(1))$, and in order for $(1,0)$ to be the identity, we can apply one of the methods to get a semifield from a pre-semifield (see [4]) and define a new multiplication. We use the same notation $\hat{\mathcal{S}}$ for the semifield with identity $(1,0)$ and multiplication

$$
(x, y) \hat{o}(u, v)=\left(x u+y \hat{f}^{-1}(1) v, x v+y \hat{f}^{-1}(1) \hat{f}(u)-y \hat{f}^{-1}(1) \hat{g}(v)\right) .
$$

Reversing this mulitplication we get the semifield $\hat{\mathcal{S}}^{*}$, i.e.,

$$
(x, y) \hat{o}^{*}(u, v)=\left(x u+y \hat{f}^{-1}(1) v, y u+v \hat{f}^{-1}(1) \hat{f}(x)-v \hat{f}^{-1}(1) \hat{g}(y)\right) .
$$



[5] G. Lunardon, Translation ovoids. Combinatorics, 2002 (Maratea), J. Geom. 76 (2003), no. 1-2, 200-215.

[6] M. Walker, A class of translation planes, Geom. Dedicata 5 (1976), 135-146.

page $7 / 7$

go back

full screen

close

quit

\section{Michel Lavrauw}

Department of Discrete Mathematics, Eindhoven University of Technology, P.O.Box 513, 5600 MB EINDHOVEN, THE NETHERLANDS,

e-mail: mlavrauw@win.tue.nl 